\title{
Marked Changes in Serum Amyloid A Distribution and High- Density Lipoprotein Structure during Acute Inflammation
}

\author{
Shitsuko Shimano, ${ }^{1}$ Ryunosuke Ohkawa ${ }^{(D},{ }^{2}$ Mayu Nambu, ${ }^{2}$ Mai Sasaoka, ${ }^{2}$ Azusa Yamazaki, ${ }^{1}$ \\ Yuki Fujii,, ${ }^{1}$ Yuna Horiuchi, ${ }^{2}$ Shao-Jui Lai, ${ }^{2}$ Takahiro Kameda, ${ }^{2}$ Naoya Ichimura, \\ Koji Fujita, ${ }^{3}$ Shuji Tohda, ${ }^{1}$ and Minoru Tozuka ${ }^{2,4}$ \\ ${ }^{1}$ Clinical Laboratory, Medical Hospital, Tokyo Medical and Dental University (TMDU), 1-5-45 Yushima, Bunkyo-ku, \\ Tokyo 113-8519, Japan \\ ${ }^{2}$ Analytical Laboratory Chemistry, Graduate School of Medical and Dental Sciences, Tokyo Medical and Dental University (TMDU), \\ 1-5-45 Yushima, Bunkyo-ku, Tokyo 113-8510, Japan \\ ${ }^{3}$ Orthopaedic and Spinal Surgery, Medical Hospital, Tokyo Medical and Dental University (TMDU), 1-5-45 Yushima, Bunkyo-ku, \\ Tokyo 113-8519, Japan \\ ${ }^{4}$ Life Science Research Center, Nagano Children's Hospital, 3100 Toyoshina, Azumino 399-8288, Japan
}

Correspondence should be addressed to Ryunosuke Ohkawa; ryu-th@umin.ac.jp

Received 23 August 2020; Revised 19 January 2021; Accepted 20 January 2021; Published 28 January 2021

Academic Editor: Andrei Surguchov

Copyright (c) 2021 Shitsuko Shimano et al. This is an open access article distributed under the Creative Commons Attribution License, which permits unrestricted use, distribution, and reproduction in any medium, provided the original work is properly cited.

\begin{abstract}
High-density lipoprotein- (HDL-) cholesterol measurements are generally used in the diagnosis of cardiovascular diseases. However, HDL is a complicated heterogeneous lipoprotein, and furthermore, it can be converted into dysfunctional forms during pathological conditions including inflammation. Therefore, qualitative analysis of pathophysiologically diversified HDL forms is important. A recent study demonstrated that serum amyloid A (SAA) can remodel HDL and induce atherosclerosis not only over long periods of time, such as during chronic inflammation, but also over shorter periods. However, few studies have investigated rapid HDL remodeling. In this study, we analyzed HDL samples from patients undergoing orthopedic surgery inducing acute inflammation. We enrolled 13 otherwise healthy patients who underwent orthopedic surgery. Plasma samples were obtained on preoperative day and postoperative days (POD) 1-7. SAA, apolipoprotein A-I (apoA-I), and apolipoprotein AII (apoA-II) levels in the isolated HDL were determined. HDL particle size, surface charge, and SAA and apoA-I distributions were also analyzed. In every patient, plasma SAA levels peaked on POD3. Consistently, the HDL apoA-I:apoA-II ratio markedly decreased at this timepoint. Native-polyacrylamide gel electrophoresis and high-performance liquid chromatography revealed the loss of small HDL particles during acute inflammation. Furthermore, HDL had a decreased negative surface charge on POD3 compared to the other timepoints. All changes observed were SAA-dependent. SAA-dependent rapid changes in HDL size and surface charge were observed after orthopedic surgery. These changes might affect the atheroprotective functions of HDL, and its analysis can be available for the qualitative HDL assessment.
\end{abstract}

\section{Introduction}

High-density lipoprotein (HDL) is a well-known, multifunctional particle that has been shown to suppress the progression of atherosclerosis by numerous epidemiological and experimental studies [1-4]. HDL and other lipoproteins transport lipids through the lymphatic and circulatory systems; many studies provided insights into the causal relation- ship between lipids and atherogenesis [5]. Consequently, HDL-cholesterol (HDL-C) measurements are generally used in the diagnosis of cardiovascular diseases, and many attempts have been made to pharmaceutically increase its levels [6-10]. However, HDL is a complicated and heterogeneous lipoprotein, and furthermore, it can be converted into dysfunctional forms during pathological conditions like diabetes $[11,12]$, oxidative stress $[13,14]$, and inflammation 
$[15,16]$. Therefore, qualitative analysis of pathophysiologically diversified HDL forms is important and has been widely conducted.

Chronic inflammation induces constant HDL remodeling that can lead to a higher risk of acute coronary syndrome. For example, levels of small HDL particles were low in patients with rheumatoid arthritis, who also had elevated coronary calcification [17]. Patients infected with human immunodeficiency virus also display increased large HDL particles and decreased small HDL particles [18].

One of the most important HDL remodeling factors is serum amyloid A (SAA). Previous studies reported that during inflammation, the blood SAA level increased up to 579$3560 \mu \mathrm{g} / \mathrm{mL}$, more than 1,000 times its basal level, following its production in the liver [19-21]. The majority of the SAA produced binds to HDL and displaces its main component, apolipoprotein A-I (apoA-I) [22-25].

Strikingly, a recent study showed that mice receiving a single injection of an adenoviral vector encoding human SAA1 displayed increased atherosclerosis, despite only brief elevations in SAA levels [26]. This suggests that SAA can have acute effects on HDL, in addition to the long-term effects observed during chronic inflammation. However, few studies have analyzed SAA distribution changes in the HDL of human subjects. In this study, we report rapid, SAA-dependent changes in HDL characteristics in patients who underwent orthopedic surgery.

\section{Materials and Methods}

2.1. Samples. Patients samples in this study were the residuals of blood samples obtained for laboratory analyses at the Clinical Laboratory of the Medical Hospital at the Tokyo Medical and Dental University. Whole blood samples were submitted to the laboratory before (PRE) and just after (POST) orthopedic surgery and on postoperative days (PODs) 1, 3, and 6 or 7 between April 2017 and March 2018. Blood samples from patients who had been diagnosed with any other medical disease (e.g., liver disease, diabetes, and other inflammatory diseases) were excluded. After anonymizing the patient samples, the untraceable blood tubes collected in vacuum tubes containing ethylenediaminetetraacetic acid dipotassium (Terumo, Tokyo, Japan) were centrifuged at $4^{\circ} \mathrm{C}$ and $2,150 \times g$ for $30 \mathrm{~min}$ for plasma collection. The samples were stored at $-80^{\circ} \mathrm{C}$ and analyzed within 4 months. Human experiments complied with all relevant national regulations and institutional policies and were performed in accordance with the tenets of the Declaration of Helsinki. The study design was disclosed publicly to patients which were offered the opportunity to opt out of this project for the use of the residual samples. The study was approved by the institutional research ethics committee of the Faculty of Medicine, Tokyo Medical and Dental University (M2016-049).

2.2. HDL Isolation. HDL $(d=1.063-1.210 \mathrm{~g} / \mathrm{mL})$ was isolated from patient plasma samples by ultracentrifugation as previously described [27]. The isolated HDL fraction was dialyzed against phosphate-buffered saline (PBS), stored at $4^{\circ} \mathrm{C}$, and used within 3 weeks.
2.3. SAA and Lipid Measurements. SAA concentrations in plasma and HDL fractions were determined using a commercially available latex agglutination-turbidimetric immunoassay kit (LZ SAA, Eiken Chemical, Tokyo, Japan). Plasma albumin and HDL-C were measured using L-Type Wako ALB-BCP and MetaboLead HDL-C kits, respectively (FUJIFILM Wako Pure Chemical Corporation, Osaka, Japan, and Kyowa Medex, Tokyo, Japan). These measurements were performed using a LABOSPECT 008 automatic analyzer (Hitachi High Technologies, Tokyo, Japan). Protein levels in HDL fractions were measured by Lowry et al.'s method [28].

2.4. Electrophoresis and Western Blot Analysis. HDL samples were resolved by sodium dodecyl sulfate- (SDS-) polyacrylamide gel (PAGE) on 16\% gels under nonreducing conditions, and by Native-PAGE on $8 \%$ gels followed by Coomassie Brilliant Blue (CBB) staining and western blot analysis, respectively, as previously described [23]. apoA-I was detected using a goat anti-apoA-I polyclonal antibody ( $1: 1,000$ dilution, Academy Bio-Medical Company, Houston, TX, USA) and horseradish peroxidase- (HRP-) conjugated rabbit anti-goat IgG $(1: 2,000$ dilution, Medical \& Biological Laboratories, Aichi, Japan), and SAA was detected using a rabbit anti-human SAA polyclonal antibody $(1: 1,200$ dilution, ASSAYPRO $\triangle \mathrm{MO}$, USA) and HRP-conjugated goat anti-rabbit IgG (1:50,000 dilution, Beckman Coulter, CA, United States). apoA-I and SAA were visualized with $3,3^{\prime}$ -diaminobenzidine- $4 \mathrm{HCl}$ and $\mathrm{H}_{2} \mathrm{O}_{2}$ or with ECL Prime Western Blotting Detection Reagent (GE Healthcare, New York, USA). Agarose electrophoresis was performed using TITAN GEL (Helena, Saitama, Japan) according to the manufacturer's protocol, and the resolved lipoproteins were stained with $0.1 \%$ Fat Red 7B. Semiquantification and analysis of the relative mobility of each band were performed by densitometry on a CS Analyzer 4 (ATTO, Tokyo, Japan).

2.5. High-Performance Liquid Chromatography (HPLC). To investigate changes in HDL particle size, patient HDL fractions were analyzed on an HPLC system equipped with a LC-20ADVP pump, a DGU-20A degassing unit, a CTO20A column oven, an SPD-20A ultraviolet detector, and an SIL-20AC autoinjector (Shimadzu Corporation, Kyoto, Japan). Each sample ( $40 \mu \mathrm{L})$ was injected into serially connected size exclusion columns (PROTEIN KW-803 and KW-804; $300 \mathrm{~mm} \times 8.0 \mathrm{~mm}$ i.d., Shodex, Tokyo, Japan), eluted with PBS at a flow rate of $1 \mathrm{~mL} / \mathrm{min}$, and monitored by absorbance at $280 \mathrm{~nm}$.

2.6. Statistical Analysis. All data represent the mean \pm standard deviation (SD) unless otherwise stated. Statistical significance was assessed using SPSS version 20.0 (IBM, Chicago, IL, USA) by Spearman's rank correlation coefficient test or unpaired Student's $t$-test. $p<0.05$ was considered statistically significant.

\section{Results}

3.1. Changes in SAA Levels before and after Surgery. SAA concentrations in patient plasma samples $(n=13)$ were determined at the PRE, POST, and POD1, 3, and 6/7 timepoints. 


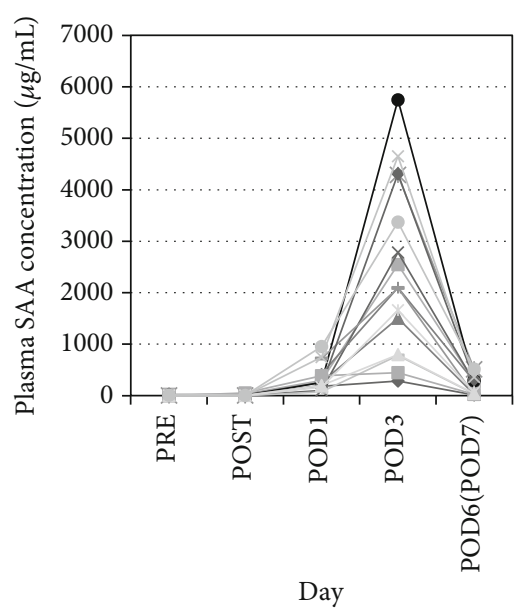

(a)

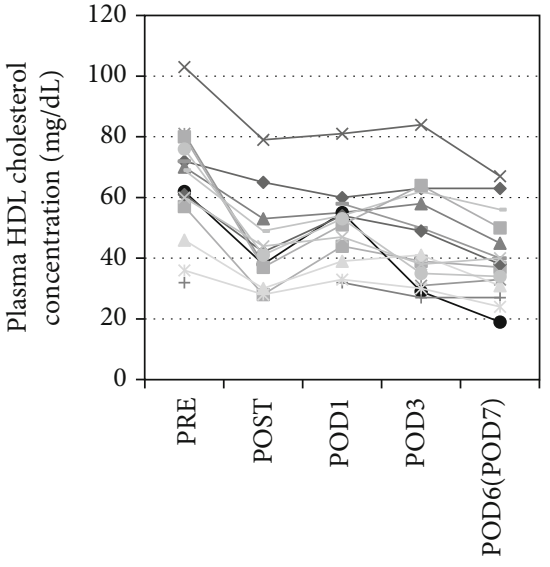

(b)

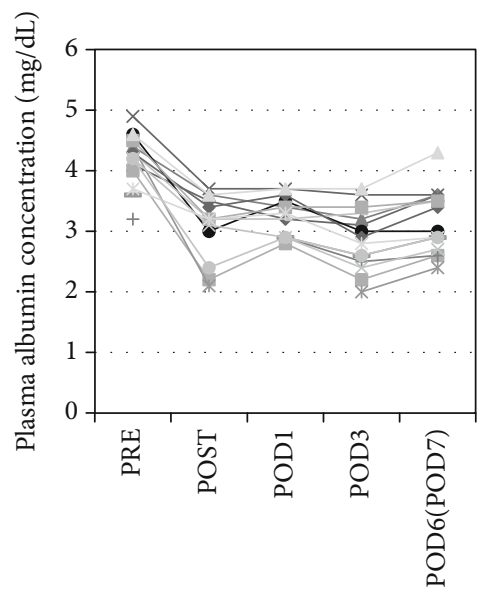

Day

(c)

FIGURE 1: Changes in plasma SAA and HDL-C levels after surgery. Plasma samples were collected from patients $(n=13)$ before (PRE) and just after (POST) orthopedic surgery and on postoperative days (POD) 1, 3, and 6 or 7. Plasma SAA (a), HDL-C (b), and albumin (c) levels were determined.

All patients except for case $2(27.7 \mu \mathrm{g} / \mathrm{mL})$ had low plasma SAA levels (mean \pm SD: $5.1 \pm 2.7 \mu \mathrm{g} / \mathrm{mL}$ ) near the reference range $(<8 \mu \mathrm{g} / \mathrm{mL}) \mathrm{PRE}$, and these values generally remained low $(3.7 \pm 2.0 \mu \mathrm{g} / \mathrm{mL})$ POST, although cases 3 and 12 displayed slight increases in SAA $(22.5$ and $53.7 \mu \mathrm{g} / \mathrm{mL}$, respectively; Figure 1(a)). Conversely, plasma SAA levels in all patients increased markedly by POD1 $(318.1 \pm 265.9 \mu \mathrm{g} / \mathrm{mL})$ and POD3 $(2552.2 \pm 1805.8 \mu \mathrm{g} / \mathrm{mL})$ and then decreased $(211.9 \pm 186.1 \mu \mathrm{g} / \mathrm{mL})$ by POD6/7. In each patient, plasma SAA peaked on POD3; however, the extent of the increase varied. Plasma HDL-C and albumin levels were also measured and displayed similar trends, decreasing by $34 \pm 14 \%$ for HDL-C and $28 \pm 12 \%$ for albumin POST compared to PRE, then remaining largely unchanged through POD3. At POD6/7, HDL-C and albumin levels decreased and increased, respectively (Figures 1 (b) and $1(\mathrm{c})$ ).

3.2. Changes in the HDL Apolipoprotein Ratio. Each HDL sample was analyzed by SDS-PAGE followed by CBB staining (Figure 2(a)). Two bands at apparent molecular masses of 28 and $8 \mathrm{kDa}$, corresponding to apoA-I and apoA-II monomers, respectively, were clearly observed in every HDL fraction at all timepoints. Bands of various intensities were also observed at $12 \mathrm{kDa}$, corresponding to SAA. Consistent with the plasma SAA level, the SAA band appeared on POD1, peaked on POD3, and was absent on POD6 (Figure 2(a)). To investigate relative changes in apolipoprotein levels, the sum of the intensities of the apoA-I, apoAII, and SAA bands was calculated at each timepoint and used to determine the intensity percentages of the individual bands. The relative intensity of the apoA-I band was significantly decreased (by $16.5 \% ; p=0.013$ ) on POD3 compared to POST and had recovered by POD6/7. Conversely, the relative amount of SAA was significantly increased (by $21.6 \%$; $p<0.001)$ on POD3 and returned to its basal level by POD6/7 (Figures 2(b) and 2(c)). However, the relative intensity changes for apoA-II did not resemble those for apoA-I. A slight decrease of $5.1 \%(p=0.01)$ was observed on POD3, which remained on POD6/7 (Figure 2(d)).

3.3. Effect of Increased SAA on HDL Particle Size. As we observed high amounts of SAA bound to HDL, we next 


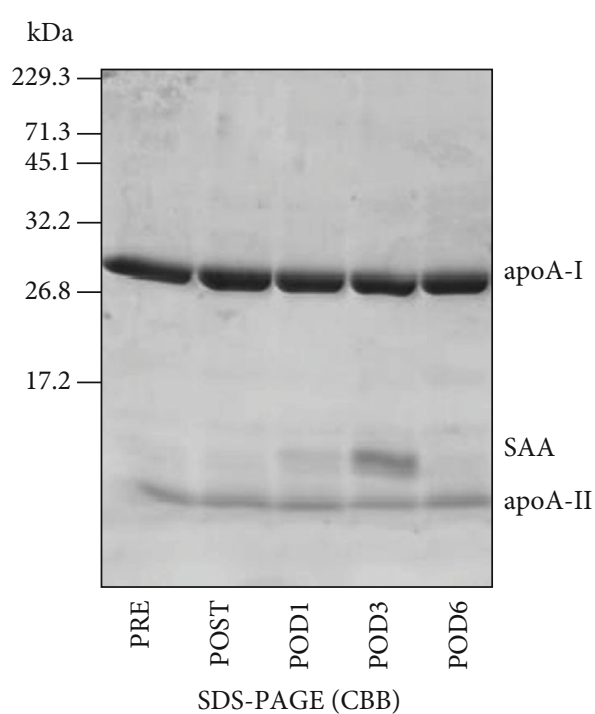

(a)

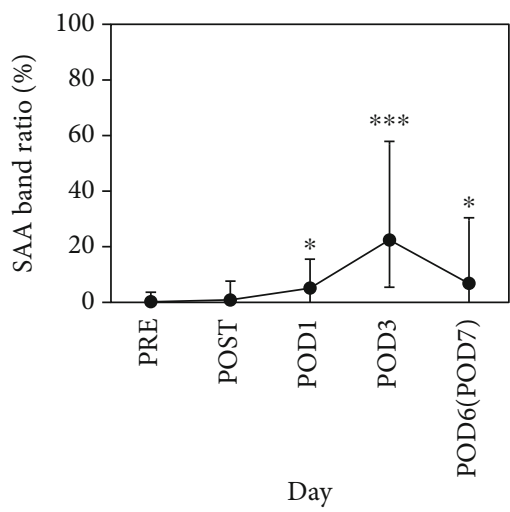

(c)

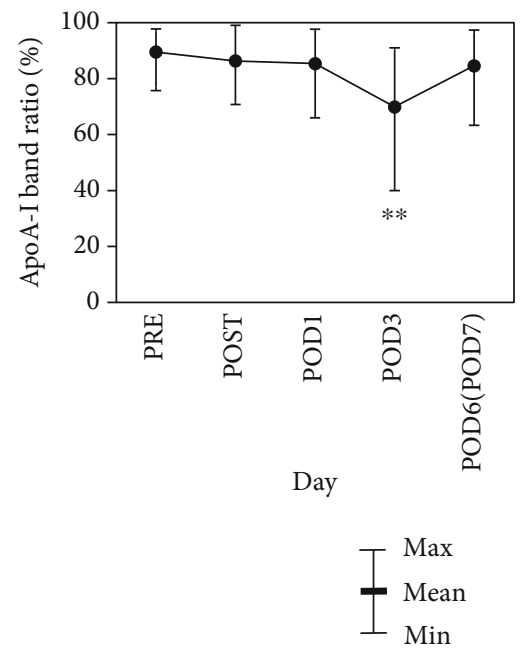

(b)

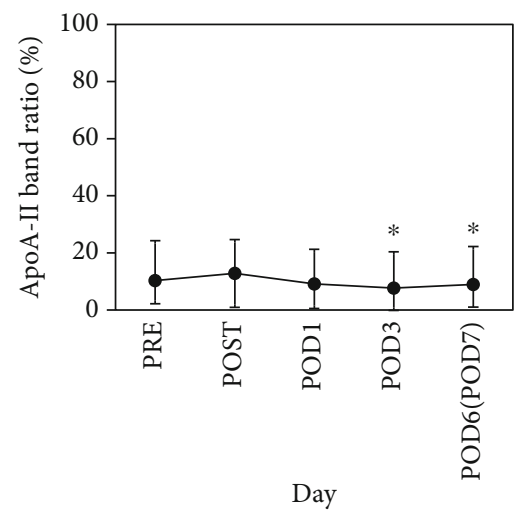

(d)

FIGURE 2: Changes in HDL apolipoprotein levels after surgery. HDL fractions were isolated from patient plasma ( $n=13)$ before (PRE) and just after (POST) orthopedic surgery and on postoperative days (POD) 1, 3, and 6 or 7 by ultracentrifugation. Fractions were resolved by SDS-PAGE ( $4 \mu \mathrm{g} / \mathrm{lane}$ ) followed by CBB staining. A representative profile is shown (a). Bands at 28, 12 , and $8 \mathrm{kDa}$ represent apoA-I, SAA, and apoA-II, respectively. Band intensities were semiquantified by densitometry. The intensities for apoA-I (b), SAA (c), and apoA-II (d) are shown as percentages of the summed intensity for all three bands. Values represent the mean, min, and max. ${ }^{*} p<0.05,{ }^{* *} p<0.005$, and ${ }^{* * *} p<0.001$ vs. POST by paired $t$-test.

examined changes in HDL particle size by HPLC. When the HPLC profiles of HDL fractions POST and on POD3 were compared, the retention time was slightly shorter on POD3, and the peak was sharper (Figure 3(a)). To investigate the association between the shortened retention time and the plasma SAA level, the ratio of the retention time on POD3 to POST was compared with the plasma SAA level on POD3. The ratio was significantly negatively correlated with the SAA level $(r=-0.611, p<0.05, n=13$; Figure 3(b)).

3.4. Changes in HDL apoA-I Distribution. Patient HDL fractions were resolved by Native-PAGE followed by western blotting for apoA-I. In PRE HDL samples, apoA-I ranged from 7.1 to $17.0 \mathrm{~nm}$ in size (Figure 4(a)). Smaller HDL particles were absent on POD3 but present on POD6/7. The percentage of decrease in HDL particle size range from POST to POD3 was compared with the POD3 plasma SAA level, and the values were significantly negatively correlated $(r=-0.787$, $p<0.005, n=13$; Figure 4(b)).

3.5. Changes in HDL SAA Distribution. Since we observed HDL particle size changes by HPLC and Native-PAGE analyses, we next examined the SAA distribution in HDL by western blot analysis. Figure 5 shows representative HDL profiles of patients with relatively low and high SAA levels on POD3 (Figures 5(a) and 5(b), respectively). After adjusting SAA levels to $500 \mu \mathrm{g} / \mathrm{lane}$, each HDL sample was resolved by Native-PAGE. In patients with low SAA, two large, noticeable bands were observed at particle sizes of 6.2 and $7.8 \mathrm{~nm}$ on POD1 and POD3 (Figure 5(a)). Conversely, in patients with high SAA, SAA was distributed on larger HDL particles on POD3, and the range of SAA distribution was wider compared to low SAA samples (Figure 5(b)). 


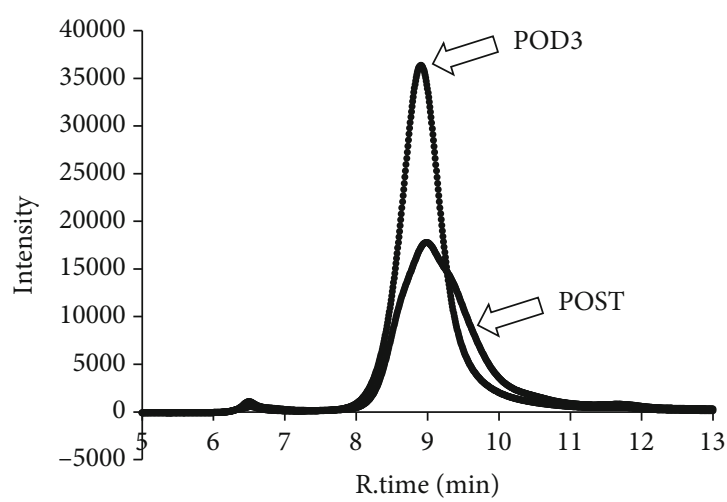

(a)

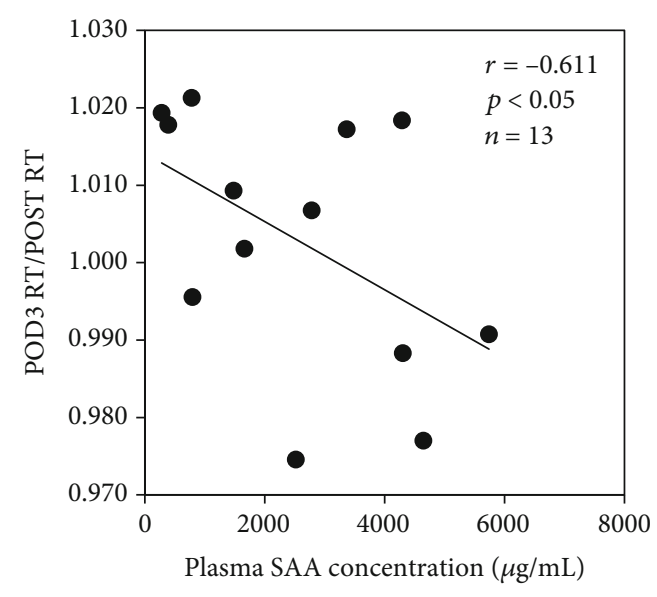

(b)

Figure 3: Association between HDL particle size and plasma SAA levels. HDL fractions from patients $(n=13)$ just after (POST) orthopedic surgery and on postoperative day 3 (POD3) were isolated by ultracentrifugation, and $40 \mu \mathrm{L}$ of $0.5 \mathrm{mg} / \mathrm{mL}$ HDL was resolved by HPLC. A representative HPLC profile is shown (a). The ratio of the retention times on POD3 and POST was compared to the plasma SAA concentration on POD3 (b). The correlation was estimated by Spearman's rank correlation coefficient test.

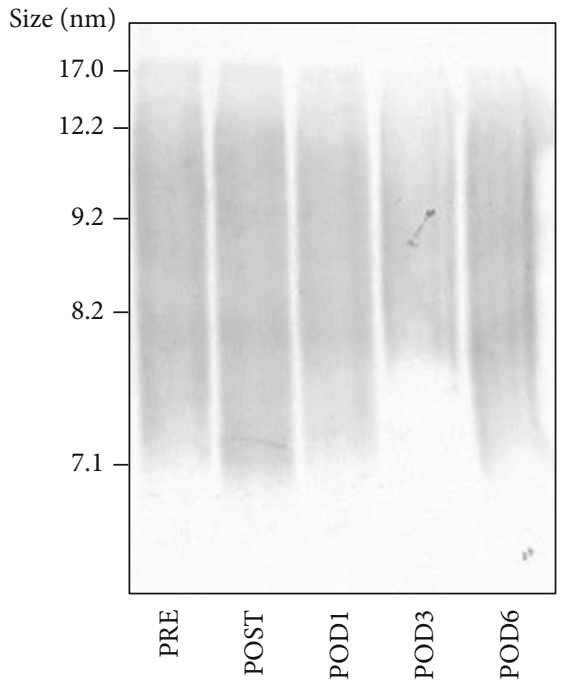

(a)

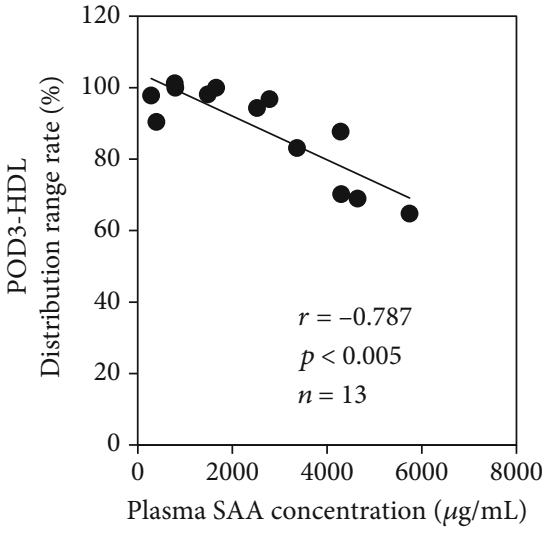

(b)

FIgURE 4: Changes in HDL apoA-I distribution after surgery. HDL fractions were isolated from patient plasma ( $n=13)$ before (PRE) and just after (POST) orthopedic surgery and on postoperative days (POD) 1, 3, and 6 or 7 by ultracentrifugation. Fractions were subjected to NativePAGE $(1.0 \mu \mathrm{g} / \mathrm{lane})$ followed by western blotting for apoA-I. A representative profile is shown (a). For each patient, the HDL particle size range was determined by densitometry and the percentage of range decrease on POD3 compared to POST was compared with the plasma SAA level on POD3 (b). The correlation was estimated by Spearman's rank correlation coefficient test.

3.6. Changes in HDL Surface Charges. The surface charges of the HDL samples were analyzed by agarose gel electrophoresis. HDL particles were observed in the alpha fraction PRE and POST; however, the relative mobility of the HDL bands was shorter on POD3 than at other timepoints (Figure 6(a)). When the distance migrated by HDL was compared with the SAA level on POD3, a significant negative correlation was observed $(r=-0.907, p<0.001, n=13$; Figure 6(b)).

\section{Discussion}

A few studies have demonstrated rapid HDL remodeling due to increased SAA in human subjects. Zimetti et al. compared the HDL of 59 subjects with acute-phase reaction (APR) related to infections, oncological causes, or autoimmune diseases and control subjects without APR and reported that in patients with APR, apoA-I-containing and medium-sized HDL particles were reduced, and HDL function was 


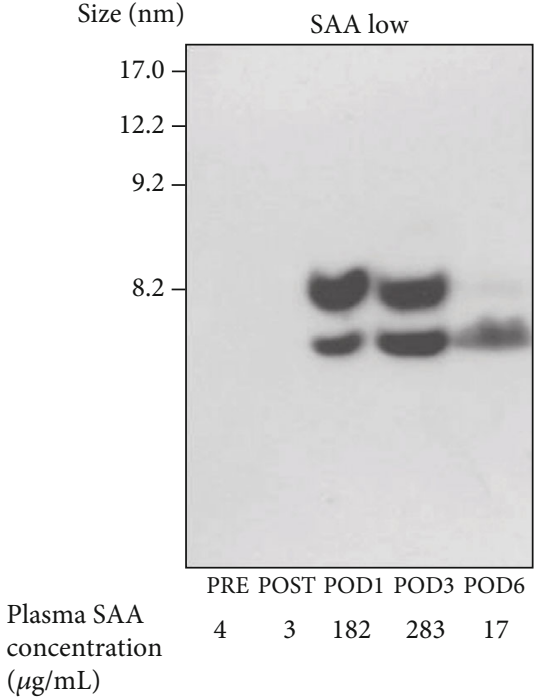

(a)

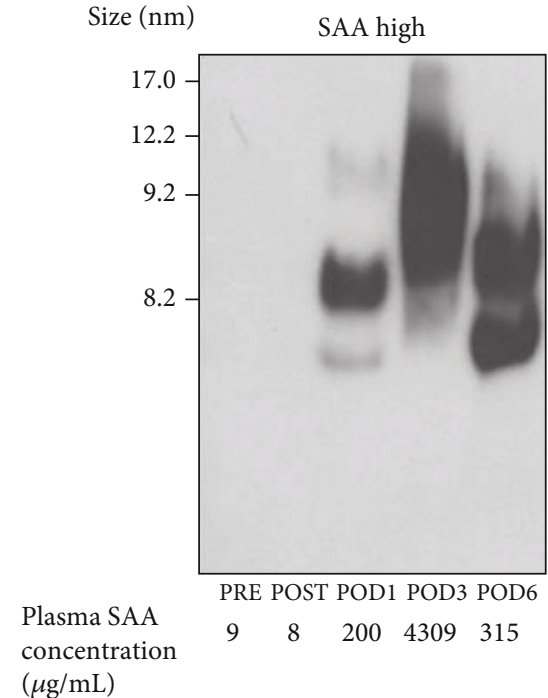

(b)

FIGURE 5: Changes in HDL SAA distribution after surgery. HDL fractions were isolated from patient plasma $(n=13)$ before (PRE) and just after (POST) orthopedic surgery and on postoperative days (POD) 1, 3, and 6 or 7 by ultracentrifugation. Fractions were subjected to NativePAGE followed by western blotting for SAA. Each sample was adjusted to $500 \mu \mathrm{g} /$ lane SAA before loading. In cases with lower SAA concentrations, the maximum volume was loaded. Representative HDL profiles from patients with low (a) and high (b) plasma SAA values are shown.

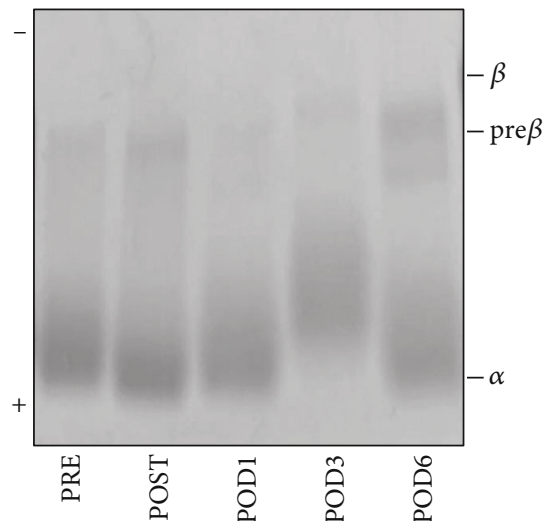

(a)

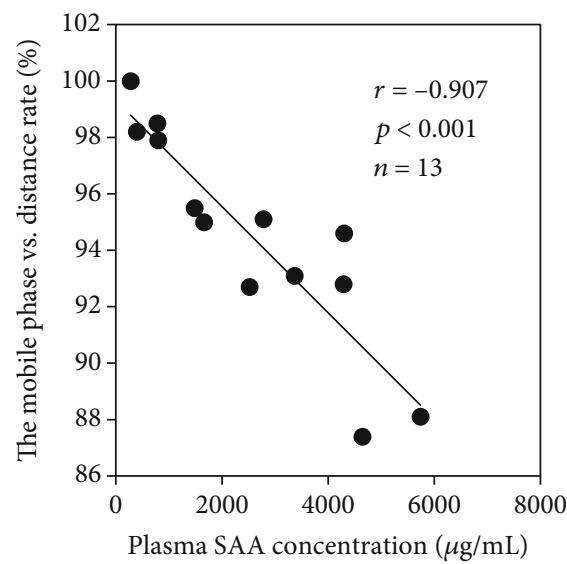

(b)

FIGURE 6: Changes in HDL surface charge after surgery. HDL fractions were isolated from patient plasma $(n=13)$ before (PRE) and just after (POST) orthopedic surgery and on postoperative days (POD) 1, 3, and 6 or 7 by ultracentrifugation. Fractions were analyzed by agarose gel electrophoresis followed by Fat Red 7B staining (HDL, $0.5 \mu \mathrm{g} / \mathrm{lane}$ ). A representative HDL profile is shown (a). The distance of HDL migration was measured by densitometry. The percentage by which migration decreased from POST to POD3 was compared with the plasma SAA level on POD3 (b). The correlation was estimated by Spearman's rank correlation coefficient test.

impaired [29]. Jahangiri et al. reported similar reductions in HDL-C and apoA-I levels, as well as decreased cholesteryl ester transfer protein levels in the HDL of patients after cardiac surgery compared to before surgery [30]. However, the heterogeneity of HDL particles observed even in healthy subjects makes it difficult to determine how SAA affects HDL structure. In addition, preexisting conditions such as coronary artery disease can make analyzing these effects even more complicated, even when comparing HDL from the same patient at different timepoints. Therefore, in this study, we analyzed HDL samples from patients undergoing orthopedic surgery who had no additional major diseases or medical issues.

SAA levels in all patients showed notable increases 3 days after surgery and decreased rapidly over the next 4 days, similar to changes reported in patients with acute inflammation [31]. In addition, HDL-C levels decreased just after surgery, before SAA levels increased. Since albumin displayed the same trend as HDL-C, these decreases were thought to be due to intravenous rehydration. Therefore, to investigate 
the effects of increased SAA on HDL, we compared HDL characteristics on POD1, 3, and 6/7 with the HDL POST, not PRE. Displacement of HDL apoA-I by SAA has been shown both in vitro and in vivo [22-24]. Consistently, reduced relative apoA-I levels were observed on POD3, with a reciprocal change observed in SAA. Conversely, only slight changes in apoA-II levels were observed. These results are consistent with previous studies on patients with severe diseases, including septicemia, septic abortion, and bacterial dysentery [22] and indicate that apoA-I release from HDL due to competition from SAA occurs in vivo.

To investigate the association between the extent of SAA binding and HDL remodeling, we analyzed changes in HDL particle size, apoA-I and SAA distributions, and surface charge. In HPLC analysis, the shorter retention time of the peak top suggested a shift toward larger HDL particles. As equal amounts of HDL were injected to the HPLC, the marked change of the sharpness of the peak also indicated a narrowed HDL particle size range. These results were consistent with the apoA-I distribution by Native-PAGE. HDL size remodeling in these patients depended on their plasma SAA levels, consistent with previous reports [22, 32]. Since large changes in HDL-C concentrations were not observed between PRE and POD3, the reduction in small HDL particles was likely not due to catabolism, but rather SAAinduced HDL enlargement. Consistent with this, SAA was mainly distributed on larger HDL particles. However, a previous study of lipopolysaccharide administration to $S A A$ knockout mice reported paradoxical results to ours, suggesting that the HDL size increase was associated with increased surface phospholipid content, not increased SAA [33]. In their Native-PAGE profile, the HDL size range, even in wild-type mice, was quite different from ours. Although additional phospholipid analysis would be instructive, this contradiction could also be simply due to differences in the organisms and inflammation induction methods used.

In our previous study, the surface charge of HDL differed between samples with low and high SAA [23]. However, these HDL samples were obtained from patients with different backgrounds (e.g., primary disease and inflammation condition), and we were unable to control for these differences. In this study, we analyzed otherwise healthy patients undergoing orthopedic surgery and observed positive changes in HDL surface charge that were SAA-dependent.

\section{Conclusions}

Our study reveals rapid apoA-I displacement, size remodeling, and surface charge changes in HDL that correspond to fluctuations in SAA levels in the same individuals at different timepoints. Recent studies from our group and others have demonstrated that SAA affects HDL functions, including its antioxidant ability $[23,34]$ and cholesterol efflux capacity $[30,35,36]$. Therefore, analysis of the SAA-specific HDL remodeling can be available for the qualitative HDL assessment as a biomarker.

\section{Data Availability}

The data used to support the findings of this study are available from the corresponding author (Ryunosuke Ohkawa, Graduate School of Medical and Dental Sciences, Tokyo Medical and Dental University (TMDU), ohkawa.alc@tmd.ac.jp) upon request.

\section{Conflicts of Interest}

The authors declared no potential conflicts of interest with respect to the research, authorship, and/or publication of this article.

\section{Acknowledgments}

This work was supported in part by Grants-in-Aid for the Encouragement of Scientists, Young Scientists (B) and Scientific Research (C) from the Japan Society for the Promotion of Science (grant numbers 16H0067 and 17H00658 to S.S. and 17K15771 and 19K07932 to R.O.).

\section{References}

[1] T. Gordon, W. P. Castelli, M. C. Hjortland, W. B. Kannel, and T. R. Dawber, "High density lipoprotein as a protective factor against coronary heart disease: the Framingham study," The American journal of medicine, vol. 62 , no. 5, pp. 707-714, 1977.

[2] P. Barter, A. M. Gotto, L. R. JC et al., "HDL cholesterol, very low levels of LDL cholesterol, and cardiovascular events," The New England Journal of Medicine, vol. 357, no. 13, pp. 1301-1310, 2007.

[3] D. J. Rader, E. T. Alexander, G. L. Weibel, J. Billheimer, and G. H. Rothblat, "The role of reverse cholesterol transport in animals and humans and relationship to atherosclerosis," Journal of Lipid Research, vol. 50, Suppl, pp. S189-S194, 2009.

[4] M. I. Mackness and P. N. Durrington, "HDL, its enzymes and its potential to influence lipid peroxidation," Atherosclerosis, vol. 115, no. 2, pp. 243-253, 1995.

[5] A. Surguchov, "Commentary: alpha-synuclein interacts with lipoproteins in plasma," Frontiers in Molecular Neuroscience, vol. 10, p. 362, 2017.

[6] M. L. Bots, F. L. Visseren, G. W. Evans et al., “Torcetrapib and carotid intima-media thickness in mixed dyslipidaemia (RADIANCE 2 study): a randomised, double-blind trial," Lancet, vol. 370, no. 9582, pp. 153-160, 2007.

[7] P. Barter, "Lessons learned from the investigation of lipid level management to understand its impact in atherosclerotic events (ILLUMINATE) trial," The American Journal of Cardiology, vol. 104, no. 10, pp. 10E-15E, 2009.

[8] G. G. Schwartz, A. G. Olsson, M. Abt et al., "Effects of dalcetrapib in patients with a recent acute coronary syndrome," The New England Journal of Medicine, vol. 367, no. 22, pp. 20892099, 2012.

[9] A. D. Mooradian and M. J. Haas, "Targeting high-density lipoproteins: increasing de novo production versus decreasing clearance," Drugs, vol. 75, no. 7, pp. 713-722, 2015.

[10] S. Choi, S. Han, S. Jeon, and D. S. Yim, "Quantitative prediction of human pharmacokinetics and pharmacodynamics of 
CKD519, a potent inhibitor of cholesteryl ester transfer protein (CETP)," Pharmaceutics, vol. 11, no. 7, 2019.

[11] S. A. Sorrentino, C. Besler, L. Rohrer et al., "Endothelial-vasoprotective effects of high-density lipoprotein are impaired in patients with type 2 diabetes mellitus but are improved after extended-release niacin therapy," Circulation, vol. 121, no. 1, pp. 110-122, 2010.

[12] C. Morgantini, A. Natali, B. Boldrini et al., "Anti-inflammatory and antioxidant properties of HDLs are impaired in type 2 diabetes," Diabetes, vol. 60, no. 10, pp. 2617-2623, 2011.

[13] T. Kameda, R. Ohkawa, K. Yano et al., "Effects of myeloperoxidase-induced oxidation on antiatherogenic functions of high-density lipoprotein," Journal of lipids, vol. 2015, Article ID 592594, 8 pages, 2015.

[14] B. Shao, M. N. Oda, J. F. Oram, and J. W. Heinecke, "Myeloperoxidase: an oxidative pathway for generating dysfunctional high-density lipoprotein," Chemical Research in Toxicology, vol. 23, no. 3, pp. 447-454, 2010.

[15] A. Jahangiri, "High-density lipoprotein and the acute phase response," Current Opinion in Endocrinology, Diabetes, and Obesity, vol. 17, no. 2, pp. 156-160, 2010.

[16] J. Watanabe, C. Charles-Schoeman, Y. Miao et al., "Proteomic profiling following immunoaffinity capture of high-density lipoprotein: association of acute-phase proteins and complement factors with proinflammatory high-density lipoprotein in rheumatoid arthritis," Arthritis and Rheumatism, vol. 64, no. 6, pp. 1828-1837, 2012.

[17] C. P. Chung, A. Oeser, P. Raggi et al., "Lipoprotein subclasses determined by nuclear magnetic resonance spectroscopy and coronary atherosclerosis in patients with rheumatoid arthritis," The Journal of Rheumatology, vol. 37, no. 8, pp. 16331638, 2010.

[18] E. Teer, D. E. Joseph, N. Driescher et al., "HIV and cardiovascular diseases risk: exploring the interplay between T-cell activation, coagulation, monocyte subsets, and lipid subclass alterations," American Journal of Physiology. Heart and Circulatory Physiology, vol. 316, no. 5, pp. H1146-H1157, 2019.

[19] J. Wilkins, J. R. Gallimore, G. A. Tennent et al., "Rapid automated enzyme immunoassay of serum amyloid a," Clinical Chemistry, vol. 40, no. 7, pp. 1284-1290, 1994.

[20] M. C. de Beer, T. Yuan, M. S. Kindy, B. F. Asztalos, P. S. Roheim, and F. C. de Beer, "Characterization of constitutive human serum amyloid A protein (SAA4) as an apolipoprotein.," Journal of Lipid Research, vol. 36, no. 3, pp. 526-534, 1995.

[21] H. Miwata, T. Yamada, M. Okada, T. Kudo, H. Kimura, and T. Morishima, "Serum amyloid A protein in acute viral infections," Archives of Disease in Childhood, vol. 68, no. 2, pp. 210214, 1993.

[22] G. A. Coetzee, A. F. Strachan, D. R. van der Westhuyzen, H. C. Hoppe, M. S. Jeenah, and F. C. de Beer, "Serum amyloid Acontaining human high density lipoprotein 3. Density, size, and apolipoprotein composition.," The Journal of Biological Chemistry, vol. 261, no. 21, pp. 9644-9651, 1986.

[23] M. Sato, R. Ohkawa, A. Yoshimoto et al., "Effects of serum amyloid $\mathrm{A}$ on the structure and antioxidant ability of highdensity lipoprotein," Bioscience Reports, vol. 36, no. 4, 2016.

[24] V. G. Cabana, N. Feng, C. A. Reardon et al., "Influence of apoA-I and apoE on the formation of serum amyloid Acontaining lipoproteins in vivo and in vitro," Journal of Lipid Research, vol. 45, no. 2, pp. 317-325, 2004.
[25] V. G. Cabana, J. R. Lukens, K. S. Rice, T. J. Hawkins, and G. S. Getz, "HDL content and composition in acute phase response in three species: triglyceride enrichment of HDL a factor in its decrease," Journal of Lipid Research, vol. 37, no. 12, pp. $2662-$ 2674, 1996.

[26] J. C. Thompson, C. Jayne, J. Thompson et al., "A brief elevation of serum amyloid A is sufficient to increase atherosclerosis[S]," Journal of Lipid Research, vol. 56, no. 2, pp. 286-293, 2015.

[27] R. J. Havel, H. A. Eder, and J. H. Bragdon, "The distribution and chemical composition of ultracentrifugally separated lipoproteins in human serum," The Journal of Clinical Investigation, vol. 34, no. 9, pp. 1345-1353, 1955.

[28] O. H. Lowry, N. J. Rosebrough, A. L. Farr, and R. J. Randall, "Protein measurement with the Folin phenol reagent," The Journal of Biological Chemistry, vol. 193, no. 1, pp. 265-275, 1951.

[29] F. Zimetti, S. De Vuono, M. Gomaraschi et al., "Plasma cholesterol homeostasis, HDL remodeling and function during the acute phase reaction[S]," Journal of Lipid Research, vol. 58, no. 10, pp. 2051-2060, 2017.

[30] A. Jahangiri, M. C. de Beer, V. Noffsinger et al., "HDL remodeling during the acute phase response," Arteriosclerosis, Thrombosis, and Vascular Biology, vol. 29, no. 2, pp. 261267, 2009.

[31] K. P. McAdam, R. J. Elin, J. D. Sipe, and S. M. Wolff, “Changes in human serum amyloid $\mathrm{A}$ and C-reactive protein after etiocholanolone-induced inflammation," The Journal of Clinical Investigation, vol. 61, no. 2, pp. 390-394, 1978.

[32] P. M. Clifton, A. M. Mackinnon, and P. J. Barter, "Effects of serum amyloid A protein (SAA) on composition, size, and density of high density lipoproteins in subjects with myocardial infarction.," Journal of Lipid Research, vol. 26, no. 12, pp. 1389-1398, 1985.

[33] M. C. de Beer, N. R. Webb, J. M. Wroblewski et al., "Impact of serum amyloid A on high density lipoprotein composition and levels," Journal of Lipid Research, vol. 51, no. 11, pp. 3117$3125,2010$.

[34] S. Jayaraman, C. Haupt, and O. Gursky, "Paradoxical effects of SAA on lipoprotein oxidation suggest a new antioxidant function for SAA[S]," Journal of Lipid Research, vol. 57, no. 12, pp. 2138-2149, 2016.

[35] D. R. van der Westhuyzen, F. C. de Beer, and N. R. Webb, "HDL cholesterol transport during inflammation," Current Opinion in Lipidology, vol. 18, no. 2, pp. 147-151, 2007.

[36] T. Vaisar, C. Tang, I. Babenko et al., "Inflammatory remodeling of the HDL proteome impairs cholesterol efflux capacity[S]," Journal of Lipid Research, vol. 56, no. 8, pp. 15191530, 2015. 